\title{
THE STRUCTURE OF SOLUTIONS \\ OF GAS DYNAMIC EQUATIONS \\ AND THE FORMATION OF THE VACUUM STATE
}

\author{
BY \\ HAITAO FAN \\ University of Wisconsin-Madison, Madison, Wisconsin
}

\begin{abstract}
The structure of the solutions of the isentropic gas dynamics equations in Eulerian coordinates given by [17] is studied. A condition under which the formation of the vacuum state occurs is obtained.
\end{abstract}

1. Introduction. One dimensional isentropic gas dynamics is described by the equations in Eulerian coordinates

$$
\begin{gathered}
\rho_{t}+(\rho u)_{x}=0 \\
(\rho u)_{t}+\left(\rho u^{2}+p(\rho)\right)_{x}=0 \quad x \in \mathbb{R}, t>0 .
\end{gathered}
$$

Here $\rho=\rho(x, t), u=u(x, t)$ are the density and the velocity of the gas, respectively. The region in the physical space where $\rho=0$ are identified with the vacuum state. The initial value problem of $(1.1 . a, b)$ and

$$
(u(x, 0), \rho(x, 0))= \begin{cases}\left(u_{-}, \rho_{-}\right) & \text {if } x<0 \\ \left(u_{+}, \rho_{+}\right) & \text {if } x>0\end{cases}
$$

is called the Riemann problem for $(1.1 \mathrm{a}, \mathrm{b})$.

The classical method of solution of the Riemann problem (1.1) is to construct the shock and wave curves for (1.1) admissible by some a-priori criterion. While many papers have been devoted to this approach (e.g. [1-9]), all these works, except [9], excluded the possiblity of the solutions with vacuum state.

Recently, Slemrod and Tzavaras [17] established the existence of solutions of (1.1) via Dafermos's variant of the vanishing viscosity approach. As was suggested and pursued in $[10-16,18]$, they replaced $(1.1 \mathrm{a}, \mathrm{b})$ by the system

$$
\begin{gathered}
\rho_{t}+(\rho u)_{x}=\epsilon t \rho_{x x}, \\
(\rho u)_{t}+\left(\rho u^{2}+p(\rho)\right)_{x}=\epsilon t(\rho u)_{x x} \quad x \in \mathbb{R}, t>0
\end{gathered}
$$

and construct their solutions as $\epsilon \rightarrow 0$ limits of solutions of (1.2), (1.1c). The advantage of this kind of "viscosity" is that it preserve the invariance of (1.1) under the transform $(x, t) \mapsto(\alpha x, \alpha t)$ where $\alpha>0$ and hence $(1.2 \mathrm{a}, \mathrm{b})$ and $(1.1 \mathrm{c})$ admits

Received December 8, 1989.

(C)1991 Brown University 
solutions of the form $\left(u_{\epsilon}(\xi), \rho_{\epsilon}(\xi)\right)$, where $\xi=\frac{x}{t}$. A simple computation shows that a classical solution of $(1.2),(1.1 \mathrm{c})$ of above form exists if and only if the following system (1.3) has a classical solution:

$$
\begin{gathered}
\epsilon \rho_{\epsilon}^{\prime \prime}=-\xi \rho_{\epsilon}^{\prime}+m_{\epsilon}^{\prime}, \\
\epsilon m_{\epsilon}^{\prime \prime}=-\xi m_{\epsilon}^{\prime}+\left(\frac{m_{\epsilon}^{2}}{\rho_{\epsilon}}+p\left(\rho_{\epsilon}\right)\right)^{\prime}, \\
\left(m_{\epsilon}(-\infty), \rho_{\epsilon}(-\infty)\right)=\left(m_{-}, \rho_{-}\right), \quad\left(m_{\epsilon}(+\infty), \rho_{\epsilon}(+\infty)\right)=\left(m_{+}, \rho_{+}\right),
\end{gathered}
$$

where $m_{\epsilon}=u_{\epsilon} \rho_{\epsilon}, m_{ \pm}=u_{ \pm} \rho_{ \pm}$and "' " is $\frac{d}{d \xi}$. In this way they reduced (1.1) to a boundary value problem for an ODE system. We summarize their results in the following theorem:

THEOREM 1.1. (a) If $p^{\prime}(\rho)>0$ for $\rho>0$ then (1.3) possesses classical solutions $\left(u_{\epsilon}(\xi), \rho_{\epsilon}(\xi)\right)$ with $\rho_{\epsilon}(\xi)>0$ for any $\epsilon>0$.

(b) If $p^{\prime}(\rho)>0$ for $\rho>0$ and either if $\int_{1}^{\infty} \frac{p^{\prime}(\rho)}{\rho} d \rho=\infty$ or if $\frac{d}{d \rho}\left(\rho^{2} p^{\prime}(\rho)\right)>0$ for $\rho>0$ and $\int_{1}^{\infty} \frac{\sqrt{p^{\prime}(\rho)}}{\rho} d \rho=\infty$, then

$$
\begin{aligned}
& T V\left(u_{\epsilon}(\xi)\right) \leq M, \\
& T V\left(\rho_{\epsilon}(\xi)\right) \leq M, \\
& T V\left(m_{\epsilon}(\xi)\right) \leq M,
\end{aligned}
$$

where $M$ is a constant independent of $\epsilon$. Therefore, there is a sequence $\left\{\epsilon_{n}\right\}$, with $\epsilon_{n} \rightarrow 0$ as $n \rightarrow \infty$, such that

$$
\left(u_{\epsilon_{n}}(\xi), \rho_{\epsilon_{n}}(\xi)\right) \rightarrow(u(\xi), \rho(\xi)) \quad \text { a.e. for } \xi \in \mathbb{R}
$$

as $n \rightarrow \infty$. Furthermore $(u(\xi), \rho(\xi))$ is a solution of (1.1).

In this paper, we study the structure of the solutions given by Slemrod and Tzavaras [17], with particular interest in the formation of the vacuum states in the solution. In what follows, the $u_{\epsilon}(\xi), \rho_{\epsilon}(\xi), u(\xi), \rho(\xi)$ are given in Theorem 1.1 above. All the $\left\{\epsilon_{n}\right\}$ in this paper are subsequences of the $\left\{\epsilon_{n}\right\}$ in Theorem 1.1(b). We further assume

Assumption 1. The function $p(\rho)$ satisfies that $p(0)=p^{\prime}(0)=0, p^{\prime}(\rho)>0$ for $\rho>0$, and $p^{\prime}(\rho)$ is increasing for $\rho \in\left(0, \delta_{1}\right)$ for some $\delta_{1}>0$.

Figure 1 gives us an idea about the function $p^{\prime}(\rho)$, where $0<\delta<\min \left(\delta_{1}, \rho_{-}, \rho_{+}\right)$ is a number such that $0<p^{\prime}(\rho)<\min _{\rho \in\left[\delta, \max \left(\rho_{-}, \rho_{+}\right)\right]}\left(p^{\prime}(\rho)\right)$ for $0<\rho<\delta$.

We notice that Assumption 1 is satisfied for the polytropic equations of state with $p(\rho)=$ Const. $\rho^{\gamma}, \gamma>1$.

The main result of this paper is the following: $(u(\xi), \rho(\xi))$ lies on a base curve in the $(u, \rho)$-plane which is differentiable with respect to $u$ or $\rho$ except possibly at an extreme point of $u$ or $\rho$. The solution consists of two wave fans of first kind and -second kind, respectively, in the sense of Dafermos [11]. $\xi$ is in the first (second) wave fan if the slope of the base curve at the points $\left(u\left(\xi_{ \pm}\right), \rho\left(\xi_{ \pm}\right)\right)$is negative (positive). $\xi$ is not a point of discontinuity of $(u(\xi), \rho(\xi))$ if $\rho(\xi-)$ or $\rho(\xi+)<\delta$. 


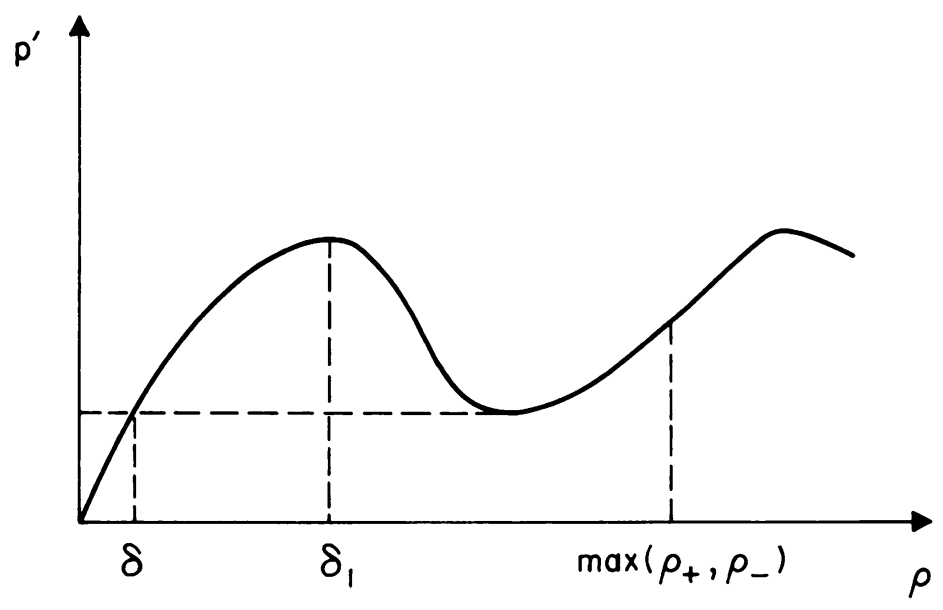

FIG. 1. The graph of $p^{\prime}(\rho)$.

Furthermore, (1.1) has at most one vacuum state which is necessarily a rarefaction one in the sense of the following definition [9]:

Definition 1.2. A vacuum state $(u(\xi), \rho(\xi)=0$ ) in a region $a<\xi<b$ (with $\rho \neq 0$ for $a-\epsilon<\xi<a$ and $b<\xi<b+\epsilon$ for some $\epsilon>0$ ) is called a rarefaction vacuum if $u(a-0)<u(b+0)$.

Moreover, if $\int_{0}^{\delta} \sqrt{p^{\prime}(\rho)} / \rho d \rho<+\infty$ and if

$$
u_{+}-u_{-}>2 \int_{0}^{\delta} \frac{\sqrt{p^{\prime}(\rho)}}{\rho} d \rho+\frac{1}{\delta} \max _{\rho \in\left[\delta, \max \left(\rho_{-}, \rho_{+}\right)\right]}\left(p^{\prime}(\rho)\right)\left(\rho_{+}+\rho_{-}-2 \delta\right),
$$

then the solution must possess a vacuum state.

The paper is divided into two sections after this one. In Sec. 2, we obtain some estimates which are of great importance to this paper. While in Sec. 3, we derive our main result.

2. Some preparations. In this section, we derive some useful lemmas. First of all, we recall Lemma 4.1 of [17] in the following lemma:

LEMMA 2.1. For the solutions of the problem (1.3) either one of the following holds:

(i) Both $u_{\epsilon}(\xi)$ and $\rho_{\epsilon}(\xi)$ are monotone.

(ii) One of the $u_{\epsilon}(\xi)$ and $\rho_{\epsilon}(\xi)$ is strictly increasing (or decreasing) while the other has exactly one critical point which is a minimum (maximum) point.

Lemma 2.2. Let $\left(u_{\epsilon}(\xi), \rho_{\epsilon}(\xi)\right)$ be a solution of $P_{\epsilon}$, then

$$
\left|\epsilon u_{\epsilon}^{\prime}(\xi)\right| \leq C_{1} \quad \text { and } \quad\left|\epsilon m_{\epsilon}^{\prime}(\xi)\right| \leq C_{1} \quad \text { for an } \xi \in \mathbb{R}
$$

where $C_{1}$ is a constant independent of $\epsilon \in(0,1)$.

Proof. For any $\xi \in \mathbb{R}, \epsilon \in(0,1)$, we can choose $\theta_{\epsilon} \in(\xi-\sqrt{\epsilon}, \xi)$ such that

$$
\left|\rho_{\epsilon}^{\prime}\left(\theta_{\epsilon}\right)\right| \leq \frac{3}{\sqrt{\epsilon}} T V\left(\rho_{\epsilon}\right) \leq \frac{3}{\sqrt{\epsilon}} M,
$$




$$
\left|m_{\epsilon}^{\prime}\left(\theta_{\epsilon}\right)\right| \leq \frac{3}{\sqrt{\epsilon}} T V\left(m_{\epsilon}\right) \leq \frac{3}{\sqrt{\epsilon}} M .
$$

Integrating from $\xi$ to $\theta_{\epsilon}$ on Eqs. $(1.3 \mathrm{a}, \mathrm{b})$, we obtain

$$
\begin{aligned}
\epsilon \rho_{\epsilon}^{\prime}(\xi)=\epsilon & \rho_{\epsilon}^{\prime}\left(\theta_{\epsilon}\right)+m_{\epsilon}(\xi)-m_{\epsilon}\left(\theta_{\epsilon}\right)-\int_{\theta_{\epsilon}}^{\xi} \zeta \rho_{\epsilon}^{\prime}(\zeta) d \zeta, \\
\epsilon m_{\epsilon}^{\prime}(\xi)= & \epsilon m_{\epsilon}^{\prime}\left(\theta_{\epsilon}\right)+m_{\epsilon}(\xi) \rho_{\epsilon}(\xi)-m_{\epsilon}\left(\theta_{\epsilon}\right) \rho_{\epsilon}\left(\theta_{\epsilon}\right) \\
& +p\left(\rho_{\epsilon}(\xi)\right)-p\left(\rho_{\epsilon}\left(\theta_{\epsilon}\right)\right)-\int_{\theta_{\epsilon}}^{\xi} \zeta m_{\epsilon}^{\prime}(\zeta) d \zeta .
\end{aligned}
$$

By employing (1.4) and (1.6), we derive that

$$
\begin{gathered}
\left.\left|\epsilon \rho_{\epsilon}^{\prime}(\xi)\right| \leq\left(5+\max \left(|\xi|,\left|\theta_{\epsilon}\right|\right)\right)\right) M, \\
\left|\epsilon m_{\epsilon}^{\prime}(\xi)\right| \leq\left(3+2 M+2 \max _{\rho \in[0, M]} p^{\prime}(\rho)+\max \left(|\xi|,\left|\theta_{\epsilon}\right|\right)\right) M .
\end{gathered}
$$

Thus, $\left|\epsilon \rho_{\epsilon}^{\prime}(\xi)\right|$ and $\left|\epsilon m_{\epsilon}^{\prime}(\xi)\right|$ are bounded uniformly in $\epsilon$ for $\xi$ in a compact subset of $\mathbb{R}$. In particular,

$$
\left|\epsilon \rho_{\epsilon}^{\prime}(0)\right| \leq C \quad \text { and } \quad\left|\epsilon m_{\epsilon}^{\prime}(0)\right| \leq C .
$$

To complete the proof, we shall prove that they are bounded in a neighborhood of $\xi= \pm \infty$.

Similar to what $[10,16,17]$ did, we rewrite Eqs. (1.3) as

$$
\epsilon y^{\prime \prime}=-\xi y^{\prime}+f(y)^{\prime}
$$

where

$$
y=\left(\begin{array}{c}
\rho_{\epsilon} \\
m_{\epsilon}
\end{array}\right), \quad f(y)=\left(\begin{array}{c}
m_{\epsilon} \\
\frac{m_{\epsilon}^{2}}{\rho_{\epsilon}}+p\left(\rho_{\epsilon}\right)
\end{array}\right) .
$$

A straightforward computation shows that

$$
\exp \left(\frac{\xi^{2}}{2 \epsilon}\right) y^{\prime}(\xi)=y^{\prime}(0)+\frac{1}{\epsilon} \int_{0}^{\xi} \nabla f(y) y^{\prime}(\zeta) \exp \left(\frac{\zeta^{2}}{2 \epsilon}\right) d \zeta .
$$

By applying the Gronwall's inequality on (2.6), we find that

$$
\left|y^{\prime}(\xi)\right| \leq\left|y^{\prime}(0)\right| \exp \left(\frac{2 R|\xi|-\xi^{2}}{2 \epsilon}\right)
$$

where $|\nabla f(y)| \leq R$ for some constant $R$, since $y$ is uniformly bounded. So,

$$
\left|y^{\prime}(\xi)\right| \leq \frac{C}{\epsilon} \exp \left(\frac{2 R|\xi|-\xi^{2}}{2 \epsilon}\right)
$$

where $C$ is the constant in (2.5). Thus, $\left|\epsilon y^{\prime}(\xi)\right|$ is uniformly bounded in $\epsilon$ for $|\xi| \geq 2 R+2$. 
LEMma 2.3. There is a subsequence of $\left\{\epsilon_{n}\right\}$, denoted by $\left\{\epsilon_{n}\right\}$ again for simplicity, such that either

$$
\varlimsup_{n \rightarrow \infty} \max \left\{\left|\frac{d u_{\epsilon_{n}}(\xi)}{d \rho_{\epsilon_{n}}(\xi)}\right| \mid \xi \in \mathbb{R}, \rho_{\epsilon_{n}}(\xi) \geq r\right\}<\infty
$$

or

$$
\varlimsup_{n \rightarrow \infty} \max \left\{\left|\frac{d \rho_{\epsilon_{n}}(\xi)}{d u_{\epsilon_{n}}(\xi)}\right| \mid \xi \in \mathbb{R}, \rho_{\epsilon_{n}}(\xi) \geq r\right\}<\infty
$$

for any $r>0$.

Proof. Suppose that (2.8a) is not true for some $r_{0}>0$. Then we have a subsequence of $\left\{\epsilon_{n}\right\}$, denoted again by $\left\{\epsilon_{n}\right\}$, such that

$$
\left|\frac{d u_{\epsilon_{n}}(\xi)}{d \rho_{\epsilon_{n}}(\xi)}\right|_{\xi=\xi_{n}} \mid \rightarrow \infty \quad \text { as } n \rightarrow \infty,
$$

with

$$
\rho_{\epsilon}\left(\xi_{n}\right) \geq r_{0}
$$

We shall show that $(2.8 \mathrm{~b})$ is true for this subsequence $\left\{\epsilon_{n}\right\}$. We claim that, for $n$ large enough, $\rho_{\epsilon_{n}}(\xi)>r_{1}=\min \left(r_{0} / 2, \rho_{-}, \rho_{+}\right)$for any $\xi \in \mathbb{R}$. Indeed, for $n$ sufficiently large,

$$
\left|\frac{d u_{\epsilon_{n}}(\xi)}{d \rho_{\epsilon_{n}}(\xi)}\right|_{\xi=\xi_{n}} \mid>C_{2}\left(r_{1}\right)
$$

where

$$
\begin{aligned}
C_{2}(r) & =\frac{C_{1}+\sqrt{C_{1}^{2}+\max _{\rho \in[r, M]} \rho^{2} p^{\prime}(\rho)}}{r^{2}} \\
& \geq \max \left\{a_{\epsilon} \pm(\xi) \mid \epsilon \in(0,1), \quad \xi \in \mathbb{R}, \text { such that } \rho_{\epsilon}(\xi) \geq r\right\}
\end{aligned}
$$

and

$$
a_{\epsilon \pm}(\xi)=\frac{\epsilon \rho_{\epsilon}^{\prime} \pm \sqrt{\left(\epsilon \rho_{\epsilon}^{\prime}\right)^{2}+\rho_{\epsilon}^{2} p^{\prime}\left(\rho_{\epsilon}\right)}}{\rho_{\epsilon}^{2}} .
$$

Without loss of generality, we can assume that

$$
\left.\frac{d u_{\epsilon_{n}}(\xi)}{d \rho_{\epsilon_{n}}(\xi)}\right|_{\xi=\xi_{n}}>C_{2}\left(r_{1}\right) .
$$

If the claim is not true, i.e.,

$$
\min _{\xi \in \mathbb{R}}\left(\rho_{\epsilon_{n}}(\xi)\right)<r_{1}
$$

for large $n$, then $\rho_{\epsilon_{n}}(\xi)$ have critical points $\xi=\tau_{n}$ which are minimum points of $\rho_{\epsilon_{n}}(\xi)$. From Eqs. (1.3), we derive that

$$
\epsilon \rho_{\epsilon} \frac{d}{d \xi}\left(\frac{d u_{\epsilon}}{d \rho}\right)=-\rho^{2}\left(\frac{d u_{\epsilon}}{d \rho}-a_{\epsilon+}(\xi)\right)\left(\frac{d u_{\epsilon}}{d \rho}-a_{\epsilon-}(\xi)\right) .
$$


An analysis on (2.12) yields that $d u_{\epsilon_{n}}(\xi) / d \rho_{\epsilon_{n}}(\xi)$ increases monotonically as $\xi$ decreases from $\xi_{n}$ until $\xi$ reaches $\xi \stackrel{=}{=} \tau_{n}$. Noting (2.9), (2.10), and (2.11), we see that

$$
\begin{aligned}
u_{\epsilon_{n}}\left(\xi_{n}\right)-u_{\epsilon_{n}}\left(\tau_{n}\right) & =\int_{\rho_{\epsilon_{n}\left(\tau_{n}\right)}}^{\rho_{\epsilon_{n}}\left(\xi_{n}\right)} \frac{d u_{\epsilon_{n}}}{d \rho} d \rho \\
& \geq\left.\left(\rho_{\epsilon_{n}}\left(\xi_{n}\right)-\rho_{\epsilon_{n}}\left(\tau_{n}\right)\right) \frac{d u_{\epsilon_{n}}(\xi)}{d \rho_{\epsilon_{n}}(\xi)}\right|_{\xi=\xi_{n}} \\
& \geq\left.\frac{r_{0} d u_{\epsilon_{n}}(\xi)}{2 d \rho_{\epsilon_{n}}(\xi)}\right|_{\xi=\xi_{n}} \rightarrow \infty \quad \text { as } n \rightarrow \infty .
\end{aligned}
$$

This contradicts the fact that $u_{\epsilon}(\xi)$ are bounded uniformly in $\epsilon$ and hence yields our claim.

If $(2.8 \mathrm{~b})$ is not true, then there is a $r_{2} \in\left(0, r_{1}\right)$ and a subsequence of $\left\{\epsilon_{n}\right\}$, denoted again by $\left\{\epsilon_{n}\right\}$, such that, for $n$ large enough,

$$
\begin{gathered}
\rho_{\epsilon_{n}}(\xi) \geq r_{1} \quad \text { for all } \xi \in \mathbb{R}, \\
\left|\frac{d u_{\epsilon_{n}}(\xi)}{d \rho_{\epsilon_{n}}(\xi)}\right|_{\xi=\zeta_{n}} \mid<C_{3}\left(r_{2}\right),
\end{gathered}
$$

for some $\zeta_{n} \in \mathbb{R}$, where

$$
\begin{aligned}
C_{3}(r) & =: \frac{\min _{\rho \in[r, M]}\left(p^{\prime}(\rho)\right)}{\sqrt{C_{1}+\max _{\rho \in[r, M]}\left(p^{\prime}(\rho) \rho^{2}\right)}+C_{1}} \\
& \leq \min \left\{a_{\epsilon} \pm(\xi) \mid \epsilon \in(0,1), \xi \in \mathbb{R}, \text { such that } \rho_{\epsilon}(\xi) \geq r\right\},
\end{aligned}
$$

where $C_{1}$ is the constant in Lemma 2.2. Equation (2.12) implies that, as $\xi$ increases, $d u_{\epsilon_{n}}(\xi) / d \rho_{\epsilon_{n}}(\xi)$ is increasing when

$$
a_{\epsilon-}(\xi)<\frac{d u_{\epsilon_{n}}(\xi)}{d \rho_{\epsilon_{n}}(\xi)}<a_{\epsilon+}(\xi)
$$

and is decreasing when

$$
\frac{d u_{\epsilon_{n}}(\xi)}{d \rho_{\epsilon_{n}}(\xi)}>a_{\epsilon+}(\xi)
$$

Similar things can be said when $\xi$ decreases. Then, (2.13) and the "initial" condition (2.14) as well as above analysis yields that

$$
\left|\frac{d u_{\epsilon_{n}}}{d \rho}\right| \leq C_{2}\left(r_{1}\right) .
$$

The contradiction between (2.15) and (2.9) completes our proof.

COROLlaRY 2.4. Let

$$
\rho_{0}=: \inf \left\{\rho_{\epsilon_{n}}(\xi) \mid \xi \in \mathbb{R}, \quad n=1,2, \ldots\right\} .
$$

If $(2.8 \mathrm{a})$ fails, then $\rho_{0}>0$.

Proof. This Corollary is the claim we proved in the proof of Lemma 2.3. 


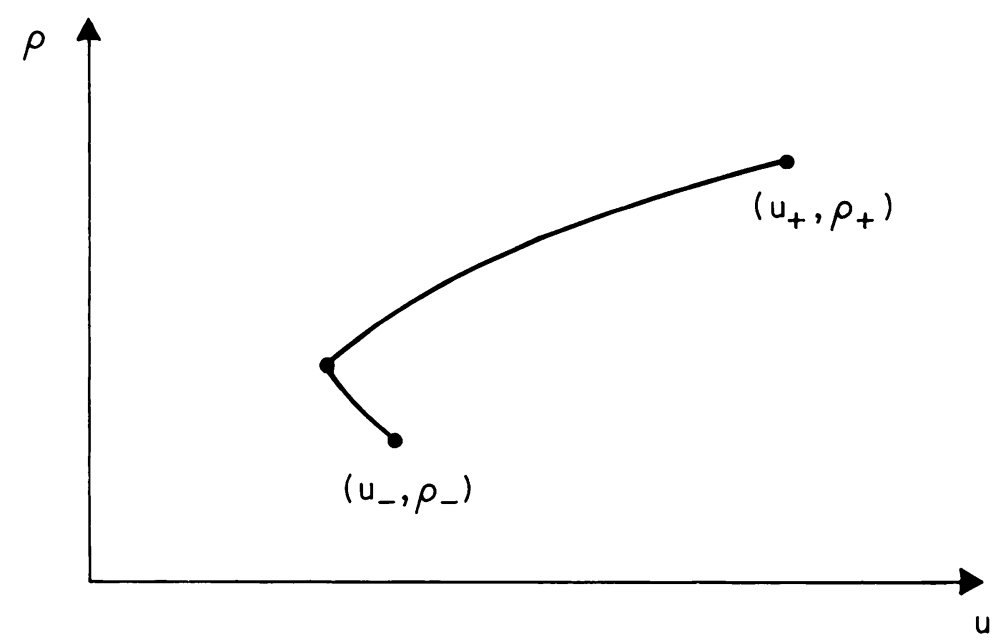

FIG. 2. The base curve for the case when $\rho_{*}>0, \rho_{+}>\rho_{-}$, $u_{+}>u_{-}$, with an extreme point for $u$ which is a minimum point.

Without loss of generality, we can assume that (2.8a) holds in the rest of this paper. Our results are valid when either of $(2.8 \mathrm{a})$ or $(2.8 \mathrm{~b})$ holds. In fact, the proofs in the case when $(2.8 \mathrm{~b})$ holds are simpler due to the absence of the vacuum state.

We use $\xi_{\epsilon_{n}}(\rho)$ to denote the inverse function of $u_{\epsilon_{n}}(\xi) . \xi_{\epsilon_{n}}(\rho)$ may have at most two continuous branches. We define

on the range of $\rho_{\epsilon_{n}}(\xi)$.

$$
\bar{u}_{\epsilon_{n}}(\rho)=: u_{\epsilon_{n}}\left(\xi_{\epsilon_{n}}(\rho)\right)
$$

$$
\begin{aligned}
& \rho_{*}=: \inf \left\{\rho_{\epsilon_{n}}(\xi) \mid \xi \in \mathbb{R}, \quad n=1,2, \ldots\right\}, \\
& \rho^{*}=: \sup \left\{\rho_{\epsilon_{n}}(\xi) \mid \xi \in \mathbb{R}, \quad n=1,2, \ldots\right\} .
\end{aligned}
$$

Without loss of generality, we can assume that $\min _{\xi \in \mathbb{R}} \rho_{\epsilon_{n}}(\xi) \rightarrow \rho_{*}$ as $n \rightarrow \infty$, and $\max _{\xi \in \mathbb{R}} \rho_{\epsilon_{n}}(\xi) \rightarrow \rho^{*}$ as $n \rightarrow \infty$. Then each $\rho \in\left(\rho_{*}, \rho^{*}\right)$ is in the domain of $\bar{u}_{\epsilon_{n}}(\rho)$ for sufficiently large $n$. Since $\left\{\bar{u}_{\epsilon_{n}}(\rho)\right\}$ satisfies (2.8a) and is uniformly bounded, we can extract a subsequence of $\left\{\epsilon_{n}\right\}$ denoted by $\left\{\epsilon_{n}\right\}$, such that $\bar{u}_{\epsilon_{n}}(\rho) \rightarrow \bar{u}(\rho)$ as $n \rightarrow \infty$ for any $\rho \in\left(\rho_{*}, \rho^{*}\right)$. Further, $\bar{u}(\rho)$ is Lipschitz on any compact subset of $\left(0, \rho^{*}\right]$. Lemma 2.1 gives us an idea about the shape of the curve of $\bar{u}(\rho)$ as drawn for the case when $u_{-}<u_{+}$in Fig. 2, and Fig. 3. We shall call this curve the base curve of the system (1.1). For convenience, we parametrize the curve by $(U(s), P(s))$, which is continuous except when $P=0$.

We summarize the above discussion in the following theorem:

THEOREM 2.5. For some sequence $\left\{\epsilon_{n}\right\}$,

$$
\bar{u}_{\epsilon_{n}}(\rho) \rightarrow \bar{u}(\rho) \text { as } n \rightarrow \infty
$$

where $\bar{u}(\rho)$ is Lipschitz on any compact subset of $\left(0, \rho^{*}\right]$. Further, $(u(\xi), \rho(\xi))$ lies on the base curve for any $\xi \in \mathbb{R}$.

Proof. The last statement follows from (2.8a). 


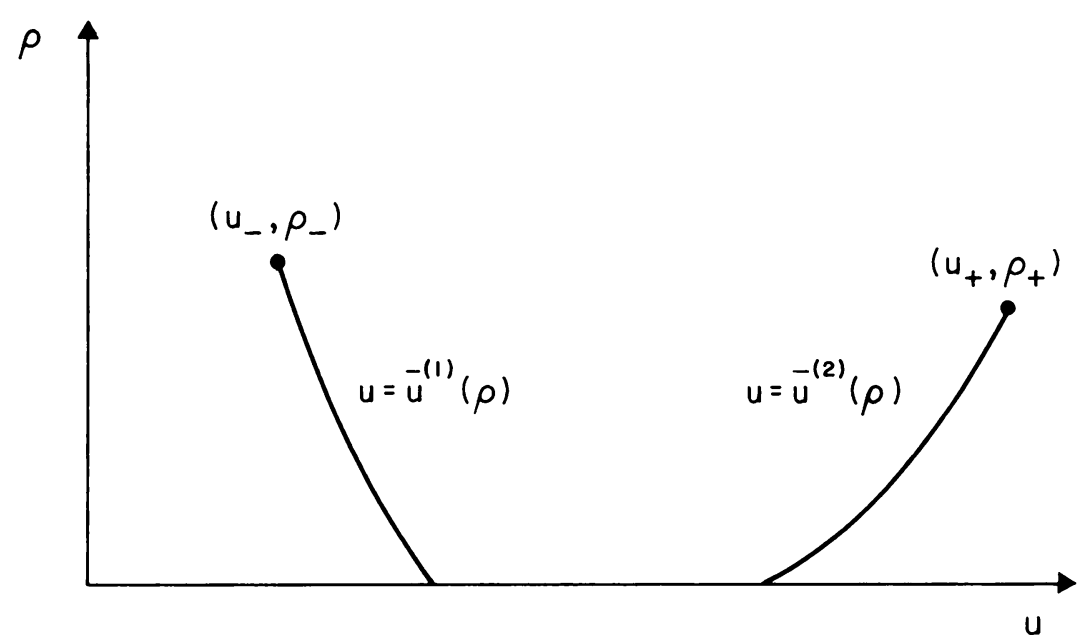

FIG. 3. The base curve $u=\bar{u}(\rho)$ for the case $\rho_{*}=0$.

3. The Structure and the vacuum state of the solution of (1.1). The study of the structure for a broad class of $2 \times 2$ hyperbolic systems including the Lagrangian isentropic gas dynamics equations via the idea of self similar boundary value problem was pioneered by Dafermos [10,11] and Dafermos and DiPerna [12]. Although his assumptions (cf. (2.1) of [11]) do not cover (1.1), the work in this section owns a sizable amount to the ideas developed in his paper [11].

We first study the discontinuities of $(u(\xi), \rho(\xi))$. Let $\xi_{0}$ be a point of discontinuity of $(u(\xi), \rho(\xi))$. By checking the Rankine-Hugoniot conditions for (1.1), we can easily prove that either $\rho\left(\xi_{0} \pm\right)=0$ or $\rho\left(\xi_{0} \pm\right) \neq 0$ holds (cf. [9]). We assume that $\rho\left(\xi_{0} \pm\right) \neq 0$. We use $C_{\xi_{0}}$ to denote the portion of the base curve connecting points $\left(u\left(\xi_{0}-\right), \rho\left(\xi_{0}-\right)\right)$ and $\left(u\left(\xi_{0}+\right), \rho\left(\xi_{0}+\right)\right)$. We choose any $(\bar{u}, \bar{\rho}) \in C_{\xi_{0}}$ with $\bar{\rho}>0$ and $\bar{\rho} \neq \rho_{*}, \rho^{*}$. We denote, by $\xi_{\epsilon_{n}}(\rho ; \bar{u}, \bar{\rho})$, the branch of the inverse function of $\rho=\rho_{\epsilon_{n}}(\xi)$ for which

$$
u_{\epsilon_{n}}\left(\xi_{\epsilon_{n}}(\bar{\rho} ; \bar{u}, \bar{\rho})\right) \rightarrow \bar{u}
$$

as $n \rightarrow \infty$. We define, for $n$ large, that

$$
\begin{gathered}
\xi_{\epsilon_{n}}=\xi_{\epsilon_{n}}(\bar{\rho})+\epsilon \zeta, \\
\hat{u}_{\epsilon_{n}}(\zeta)=u_{\epsilon_{n}}\left(\xi_{\epsilon_{n}}\right), \\
\hat{\rho}_{\epsilon_{n}}(\zeta)=\rho_{\epsilon_{n}}\left(\xi_{\epsilon_{n}}\right) .
\end{gathered}
$$

LEMMA 3.1. Let $\xi_{0}$ be a point of discontinuity of $(u(\xi), \rho(\xi))$ with $\rho\left(\xi_{0} \pm\right)>0$. For $\left(\hat{u}_{\epsilon_{n}}(\zeta), \hat{\rho}_{\epsilon_{n}}(\zeta)\right)$ defined above with $\bar{\rho}>0$, there is a subsequence of $\left\{\epsilon_{n}\right\}$, denoted by $\left\{\epsilon_{n}\right\}$ for simplicity, such that

$$
\left(\hat{u}_{\epsilon_{n}}(\zeta), \hat{\rho}_{\epsilon_{n}}(\zeta)\right) \rightarrow(\hat{u}(\zeta), \hat{\rho}(\zeta)) \in C^{1}\left(\mathbb{R} ; \mathbb{R}^{2}\right) \quad \text { as } n \rightarrow \infty
$$


uniformly for $\zeta$ in a compact subset of $\mathbb{R}$. $(\hat{u}(\zeta), \hat{\rho}(\zeta))$ satisfies the following initial value problem:

$$
\begin{gathered}
\frac{d \hat{\rho}(\zeta)}{d \zeta}=\hat{m}(\zeta)-m\left(\xi_{0}-\right)-\xi_{0}\left(\hat{\rho}(\zeta)-\rho\left(\xi_{0}-\right)\right), \\
\frac{d \hat{m}(\zeta)}{d \zeta}=\hat{u}(\zeta) \hat{m}(\zeta)-m\left(\xi_{0}-\right) u\left(\xi_{0}-\right) \\
-\xi_{0}\left(\hat{m}(\zeta)-m\left(\xi_{0}-\right)\right)+p(\hat{\rho}(\zeta))-p\left(\rho\left(\xi_{0}-\right)\right), \\
\hat{\rho}(0)=\bar{\rho}, \quad \hat{u}(0)=\bar{u},
\end{gathered}
$$

where $\hat{m}(\zeta)=\hat{u}(\zeta) \hat{\rho}(\zeta)$. Furthermore, $(\hat{u}(\zeta), \hat{\rho}(\zeta))$ lies on $C_{\xi_{0}}$, if $\hat{\rho}(\zeta)>0$.

Proof. Clearly, $\left(\hat{u}_{\epsilon_{n}}(\zeta), \hat{\rho}_{\epsilon_{n}}(\zeta)\right)$ are of uniformly bounded total variation since $\left(u_{\epsilon}(\xi), \rho_{\epsilon}(\xi)\right)$ are so. Thus, there is a subsequence of $\left\{\epsilon_{n}\right\}$, denoted by $\left\{\epsilon_{n}\right\}$ again, such that

$$
\left(\hat{u}_{\epsilon_{n}}(\zeta), \hat{\rho}_{\epsilon_{n}}(\zeta)\right) \rightarrow(\hat{u}(\zeta), \hat{\rho}(\zeta))
$$

as $n \rightarrow \infty$, for any $\zeta \in \mathbb{R}$.

Let $V_{\xi_{0}}$ be a neighborhood of $\left(u\left(\xi_{0}-\right), \rho\left(\xi_{0}-\right)\right)$ in the $(u, \rho)$-plane small enough such that $V_{\xi_{0}}$ is bounded away from the $\rho$-axis. Choose

$$
\left(u_{\delta}, \rho_{\delta}\right) \in C_{\xi_{0}} \cap V_{\xi_{0}}
$$

close enough to $\left(u\left(\xi_{0}\right), \rho\left(\xi_{0}\right)\right)$ such that the portion of $C_{\xi_{0}}$ connecting $\left(u\left(\xi_{0}\right), \rho\left(\xi_{0}\right)\right.$ and $\left(u_{\delta}, \rho_{\delta}\right)$ is in $V_{\xi_{0}}$. Without loss of generality, we can assume this portion is on the branch $u=\bar{u}^{(1)}(\rho)$. There is a

$$
\theta_{\epsilon_{n}} \in\left(\xi_{\epsilon_{n}}\left(\rho_{\delta}\right)-\sqrt{\epsilon_{n}}, \xi_{\epsilon_{n}}\left(\rho_{\delta}\right)\right)
$$

such that

$$
\begin{aligned}
\left|u_{\epsilon_{n}}^{\prime}\left(\theta_{\epsilon_{n}}\right)\right| & \leq \frac{3}{\sqrt{\epsilon_{n}}} T V\left(u_{\epsilon}\right) \leq \frac{3 M}{\sqrt{\epsilon_{n}}}, \\
\left|\rho_{\epsilon_{n}}^{\prime}\left(\theta_{\epsilon_{n}}\right)\right| & \leq \frac{3}{\sqrt{\epsilon_{n}}} T V\left(\rho_{\epsilon}\right) \leq \frac{3 M}{\sqrt{\epsilon_{n}}} .
\end{aligned}
$$

From (3.9) and

$$
\left(u_{\epsilon_{n}}(\xi), \rho_{\epsilon_{n}}(\xi)\right) \rightarrow(u(\xi), \rho(\xi))
$$

it is easily seen that

$$
\xi_{\epsilon_{n}}\left(\rho_{\delta}\right) \rightarrow \xi_{0}
$$

and hence $\theta_{\epsilon_{n}} \rightarrow \xi_{0}$. Since (2.8a) holds, $\rho_{\epsilon_{n}}(\xi)$ is monotone. So, $\liminf _{n \rightarrow \infty} \rho_{\epsilon_{n}}\left(\theta_{\epsilon_{n}}\right)$ is between $\rho\left(\xi_{0}-\right)$ and $\rho_{\delta}$. Thus, without loss of generality,

$$
\rho_{\epsilon_{n}}\left(\theta_{\epsilon_{n}}\right) \rightarrow \rho_{1} \quad \text { as } n \rightarrow \infty
$$

for some $\rho_{1}$ between $\rho\left(\xi_{0}-\right)$ and $\rho \delta$. Then we have by $(2.8 \mathrm{a})$ that

$$
\lim _{n \rightarrow \infty} \bar{u}_{\epsilon_{n}}^{(1)}\left(\rho_{\epsilon_{n}}\left(\theta_{\epsilon_{n}}\right)\right)=\bar{u}^{(1)}\left(\rho_{1}\right) \text {. }
$$


In the rest of this paper, we shall write $\epsilon$ instead of $\epsilon_{n}$ for simplicity. Integrating Eqs. (1.3) from $\theta_{e}$ to $\tau_{\epsilon}=: \xi_{\epsilon}(\bar{\rho})+\epsilon \zeta$, we get

$$
\begin{gathered}
\frac{d \hat{\rho}_{\epsilon}(\zeta)}{d \zeta}=\hat{m}_{\epsilon}(\zeta)-m_{\epsilon}\left(\theta_{\epsilon}\right)-\xi_{0}\left(\hat{\rho}_{\epsilon}(\zeta)-\rho_{\epsilon}\left(\theta_{\epsilon}\right)\right)+\epsilon \rho_{\epsilon}^{\prime}\left(\theta_{e}\right) \\
\quad-\int_{\theta_{e}}^{\tau_{\epsilon}}\left(\zeta-\xi_{0}\right) \rho_{\epsilon}^{\prime}(\zeta) d \zeta \\
\frac{d \hat{m}_{\epsilon}(\zeta)}{d \zeta}=\hat{m}_{\epsilon}\left(\zeta \hat{u}_{\epsilon}(\zeta)-m_{\epsilon}\left(\theta_{e}\right) u_{\epsilon}\left(\theta_{e}\right)-\xi_{0}\left(\hat{m}_{\epsilon}(\zeta)-m_{\epsilon}\left(\theta_{e}\right)\right)\right. \\
+p\left(\hat{\rho}_{\epsilon}(\zeta)\right)-p\left(\rho_{\epsilon}\left(\theta_{\epsilon}\right)\right)+\epsilon m_{\epsilon}^{\prime}\left(\theta_{e}\right)-\int_{\theta_{\epsilon}}^{\tau_{\epsilon}}\left(\zeta-\xi_{0}\right) m_{\epsilon}^{\prime}(\zeta) d \zeta .
\end{gathered}
$$

By (3.11) the second last terms in (3.15) approach to 0 as $n \rightarrow \infty$ uniformly for $\zeta$. Recalling (1.4) and that $\theta_{\epsilon} \rightarrow \xi_{0}, \tau_{\epsilon} \rightarrow \xi_{0}$ as $n \rightarrow \infty$ uniformly in $\zeta$ for $\zeta$ in compact subsets of $\mathbb{R}$, we see that the last terms in (3.15) vanish, as $n \rightarrow \infty$, uniformly for $\zeta$ in a compact set. A classical theorem of the theory of ODE says that $\left(\hat{u}_{\epsilon}(\zeta), \hat{\rho}_{\epsilon}(\zeta)\right) \rightarrow(\hat{u}(\zeta), \hat{\rho}(\zeta))$ as $n \rightarrow \infty$ uniformly on compact subsets of $\mathbb{R}$, and that

$$
\begin{gathered}
\frac{d \hat{u}(\zeta)}{d \zeta}=\hat{m}(\zeta)-\bar{u}^{(1)}\left(\rho_{1}\right) \rho_{1}-\xi_{0}\left(\hat{\rho}(\zeta)-\rho_{1}\right), \\
\frac{d \hat{m}(\zeta)}{d \zeta}=\hat{m}(\zeta) \hat{u}(\zeta)-\bar{u}^{(1) 2}\left(\rho_{1}\right) \rho_{1}-\xi_{0}\left(\hat{m}(\zeta)-\bar{u}^{(1)}\left(\rho_{1}\right) \rho_{1}\right) \\
+p(\hat{\rho}(\zeta))-p\left(\rho_{1}\right), \\
\hat{\rho}(0)=\bar{\rho}, \\
\hat{u}(0)=u^{(1)}(\bar{\rho})=\bar{u} .
\end{gathered}
$$

By letting $V_{\xi_{0}}$ shrink to $\left(u\left(\xi_{0}-\right), \rho\left(\xi_{0}-\right)\right)$ to force $\left(\bar{u}^{(1)}\left(\rho_{1}\right), \rho_{1}\right) \rightarrow\left(u\left(\xi_{0}-\right), \rho\left(\xi_{0}-\right)\right)$, we obtain (3.6) and (3.7). The last statement is an immediate consequence of (2.8a) and the uniqueness of (3.6), (3.7).

REMARK. From (3.6), we can derive an equation for $\hat{u}(\zeta)$ :

$$
\begin{aligned}
\hat{\rho}(\zeta) \frac{d \hat{u}(\zeta)}{d \zeta}= & \left(\hat{u}(\zeta)-u\left(\xi_{0}-\right)\right)\left(u\left(\xi_{0}-\right)-\xi_{0}\right) \rho\left(\xi_{0}-\right) \\
& +p(\hat{\rho}(\zeta))-p\left(\rho\left(\xi_{0}-\right)\right) .
\end{aligned}
$$

LEMMA 3.2. Let $\xi_{0}$ be a point of discontinuity of $(u(\xi), \rho(\xi))$. Then for any $(\tilde{u}, \tilde{\rho}) \in C_{\xi_{0}}$ with $\tilde{p}>0$ and $\tilde{p} \neq \rho_{*}, \rho^{*}$, we have that

(a) if $P(s)$ is increasing (decreasing) at $(\tilde{u}, \tilde{\rho})$, then

$$
\tilde{u} \tilde{\rho}-m\left(\xi_{0}-\right)-\xi_{0}\left(\tilde{\rho}-\rho\left(\xi_{0}-\right)\right) \geq 0 \quad(\leq 0) ;
$$

(b) If $U(s)$ is increasing (decreasing) at $(\tilde{u}, \tilde{\rho})$, then

$$
\left(\tilde{u}-u\left(\xi_{0}-\right)\right)\left(u\left(\xi_{0}-\right)-\xi_{0}\right) \rho\left(\xi_{0}-\right)+p(\tilde{\rho})-p\left(\rho\left(\xi_{0}-\right)\right) \geq 0 \quad(\leq 0) ;
$$

(c) (3.19a) holds as an equality if and only if $(3.19 b)$ holds as an equality;

(d) if either one of $(3.19 \mathrm{a}, \mathrm{b})$ holds as an equality, then

$$
\tilde{u}^{2} \tilde{\rho}-m\left(\xi_{0}-\right) u\left(\xi_{0}-\right)-\xi_{0}\left(\tilde{u} \tilde{\rho}-m\left(\xi_{0}-\right)\right)+p(\tilde{\rho})-p\left(\rho\left(\xi_{0}-\right)\right)=0 .
$$


Proof. (a) Let $(\hat{u}(\zeta), \hat{\rho}(\zeta))$ be the solution (3.6), (3.7) with $(\bar{u}, \bar{\rho})=(\tilde{u}, \tilde{\rho})$. (3.19a) follows from (3.6a) and the fact that $\hat{\rho}(\zeta)$ is increasing (decreasing) at $\zeta=0$ if $P(s)$ is increasing (decreasing) at $(\tilde{u}, \tilde{\rho})$. (b) can be proved similarly.

(c) We see from (3.6a) that (3.19a) holds as an equality if and only if $\left.\frac{d \hat{\rho}(\zeta)}{d \zeta}\right|_{\zeta=0}=0$. We take $\frac{d}{d \zeta}$ on Eqs. (3.6) to obtain that

$$
\begin{gathered}
\frac{d^{2} \hat{\rho}(\zeta)}{d^{2} \zeta}=\hat{u}(\zeta) \frac{d \hat{\rho}(\zeta)}{d \zeta}+\hat{\rho}(\zeta) \frac{d \hat{u}(\zeta)}{d \zeta}-\xi_{0} \frac{d \hat{\rho}(\zeta)}{d \zeta} \\
\hat{\rho}(\zeta) \frac{d^{2} \hat{u}(\zeta)}{d^{2} \zeta}+\frac{d \hat{\rho}(\zeta)}{d \zeta} \frac{d \hat{u}(\zeta)}{d \zeta} \\
\quad=\rho\left(\xi_{0}-\right)\left(u\left(\xi_{0}-\right)-\xi_{0}\right) \frac{d \hat{u}(\zeta)}{d \zeta}+p^{\prime}(\hat{\rho}(\zeta)) \frac{d \hat{\rho}(\zeta)}{d \zeta}
\end{gathered}
$$

Since $\bar{\rho} \neq \rho_{*}, \rho^{*}, \bar{\rho}$ is not an extreme value for $\hat{\rho}(\zeta)$, it must be an inflection point and hence $\left.\left(d^{2} \hat{\rho}(\zeta) / d^{2} \zeta\right)\right|_{\zeta=0}=0$. It immediately follows from (3.21) that $d \hat{\rho}(\zeta) /\left.d \zeta\right|_{\zeta=0}=0$ if and only if $d \hat{u}(\zeta) /\left.d \zeta\right|_{\zeta=0}=0$.

(d) is obvious.

We are interested in the differentiablity of the function $\bar{u}(\rho)$. Since the base curve $(U(s), P(s))$ is oriented in the direction in which $s$ increases, we can talk about the "right" and "left" sides of $\left(U\left(s_{0}\right), P\left(s_{0}\right)\right)$ by which we mean the portions of the curve with $s<s_{0}$ and $s>s_{0}$, respectively. Accordingly, we can define the "left" derivative

$$
\frac{d \bar{u}(\rho)}{d^{(-)} \rho}=\left.\frac{d U(s)}{d P(s)}\right|_{s=s_{0}-}
$$

and "right" derivatives

$$
\frac{d \bar{u}(\rho)}{d^{(+)} \rho}=\left.\frac{d U(s)}{d P(s)}\right|_{s=s_{0}+}
$$

of $\bar{u}(\rho)$ at the point $(u, \rho)=\left(U\left(s_{0}\right), P\left(s_{0}\right)\right)$. We define

$$
\begin{aligned}
& S(U(s), P(s))= \begin{cases}1 & \text { if both } U(s) \text { and } P(s) \text { are strictly increasing } \\
& \text { or strictly decreasing at } s, \\
-1 & \text { if both } U(s) \text { and }-P(s) \text { are strictly increasing } \\
\text { or strictly decreasing at } s, \\
0 \quad & \text { otherwise, }\end{cases} \\
& S\left(U\left(s_{0}\right), P\left(s_{0}\right) ;+\right)=\lim _{s \rightarrow s_{0}+} S(U(s), P(s)), \\
& S\left(U\left(s_{0}\right), P\left(s_{0}\right) ;-\right)=\lim _{s \rightarrow s_{0}-} S(U(s), P(s)) .
\end{aligned}
$$

LemMA 3.3. Let $\xi_{0}$ be a point of discontinuity of $(u(\xi), \rho(\xi))$ with $\rho\left(\xi_{0} \pm\right)>0$ for any $(\tilde{u}, \tilde{\rho}) \in C_{\xi_{0}}$.

(a) if $(3.19 \mathrm{a})$ or $(3.19 \mathrm{~b})$ holds as a strict inequality, then

$$
\left.\tilde{\rho} \frac{d \bar{u}(\rho)}{d \rho}\right|_{(\bar{u}(\rho), \rho)=(\tilde{u}, \tilde{\rho})}=\frac{\left(\tilde{u}-u\left(\xi_{0}-\right)\right)\left(m\left(\xi_{0}-\right)-\xi_{0} \rho\left(\xi_{0}-\right)\right)+p(\tilde{\rho})-p\left(\rho\left(\xi_{0}-\right)\right)}{\tilde{m}-m\left(\xi_{0}-\right)-\xi_{0}\left(\tilde{\rho}-\rho\left(\xi_{0}-\right)\right)} .
$$


(b) If (3.19a) or (3.19b) holds as an equality, then

$$
\left.\frac{d \bar{u}(\rho)}{d \rho}\right|_{(\bar{u}(\rho), \rho)=(\tilde{u}, \tilde{\rho})}=S(\tilde{u}, \tilde{\rho}) \frac{\sqrt{p^{\prime}(\tilde{\rho})}}{\tilde{\rho}} .
$$

(c) The "right" derivative of $\bar{u}(\rho)$ at $\left(u\left(\xi_{0}-\right), \rho\left(\xi_{0}-\right)\right)$ is

$$
S\left(u\left(\xi_{0}-\right), \rho\left(\xi_{0}-\right) ;+\right) \frac{\sqrt{p^{\prime}\left(\rho\left(\xi_{0}-\right)\right)}}{\rho\left(\xi_{0}-\right)} .
$$

The "left" derivative of $\bar{u}(\rho)$ at $\left(u\left(\xi_{0}+\right), \rho\left(\xi_{0}+\right)\right)$ is

$$
S\left(u\left(\xi_{0}+\right), \rho\left(\xi_{0}+\right) ;-\right) \frac{\sqrt{p^{\prime}\left(\rho\left(\xi_{0}+\right)\right)}}{\rho\left(\xi_{0}+\right)} .
$$

Proof. (a) follows from (3.6a) and (3.18).

(b) Assume that Eqs. (3.19) hold as equalities at $(\tilde{u}, \tilde{\rho})$ with $\tilde{\rho}>0$. Since $\tilde{p} \neq \rho_{*}, \rho^{*}, U(s)$ and $P(s)$ are monotone near the point $(\tilde{u}, \tilde{\rho})$. For definiteness, we assume that $P(s)$ is decreasing and $U(s)$ is increasing there and $(\tilde{u}, \tilde{\rho})$ is on the branch (if applicable) $\bar{u}^{(1)}(\rho)$. The proofs for other cases are similar. We shall show that $\bar{u}^{(1)}(\rho)$ has "left" derivative as $(\tilde{u}, \tilde{\rho})$ given by $(3.23)$.

CASE 1. (3.19a) holds as a strict inequality for any $\bar{\rho} \in\left(\tilde{\rho}, \rho_{1}\right)$ for some $\rho_{1}$.

Let $(\hat{u}(\zeta), \hat{\rho}(\zeta))$ be the solution of $(3.6)$ with initial value $(\hat{u}(0), \hat{\rho}(0))=$ $\left(\bar{u}^{(1)}\left(\rho_{1}\right), \rho_{1}\right)$. Then it is obvious that

$$
(\hat{u}(\zeta), \hat{\rho}(\zeta)) \rightarrow(\tilde{u}, \tilde{\rho}) \quad \text { as } \zeta \rightarrow \infty .
$$

With the help of (3.6), we obtain

$$
\begin{aligned}
\frac{d}{d \zeta}\left(\frac{\bar{u}^{(1)}(\rho)}{d \rho}\right) & =\frac{d}{d \zeta}\left(\frac{d \hat{u}(\zeta)}{d \zeta} / \frac{d \hat{\rho}(\zeta)}{d \zeta}\right) \\
& =\left(\frac{d \bar{u}^{(1)}(\rho)}{d \rho}-a_{\epsilon+}(\zeta)\right)\left(\frac{d \bar{u}^{(1)}(\rho)}{d \rho}-a_{\epsilon_{-}}(\zeta)\right)
\end{aligned}
$$

where

$$
a_{\epsilon \pm}(\zeta)=: \frac{-\hat{\rho}^{\prime}(\zeta) \pm \sqrt{\hat{\rho}^{\prime 2}(\zeta)+\hat{\rho}(\zeta)^{2} p^{\prime}(\hat{\rho}(\zeta))}}{\hat{\rho}(\zeta)^{2}} \gtrless 0
$$

We claim that

$$
\min _{\hat{\rho}(\zeta) \in\left[\tilde{\rho}, \rho_{1}\right]} a_{\epsilon_{-}}(\zeta) \leq \frac{d \overline{\mathcal{u}}^{(1)}(\rho)}{d \rho} \leq \max _{\hat{\rho}(\zeta) \in\left[\hat{\rho}, \rho_{1}\right]} a_{\epsilon_{-}}(\zeta) .
$$

An analysis on (3.26) similar to what we did for (2.12) shows that if the claim is not true, either $\left.d \bar{u}^{(1)}(\rho) / d \rho\right) \rightarrow-\infty$ as $\zeta \rightarrow+\infty$ or $d \bar{u}^{(1)}(\rho) / d \rho>0$ for $\zeta$ large will hold. But none of them is possible because the former contradicts the fact that $\bar{u}^{(1)}(\rho)$ is Lipschitzian for $\rho \geq r$ for any $r>0$, while the latter violates our assumption that 
$U(s),-P(s)$ are increasing on the portion of $C_{\xi_{0}}$ from $\left(u_{1}, \rho_{1}\right)$ to $(\tilde{u}, \tilde{\rho})$, which is made at the beginning of this proof. By letting $\rho_{1} \rightarrow \tilde{\rho}+$ in (3.28), we obtain that

$$
\frac{d \bar{u}^{(1)}(\rho)}{d \rho}=\frac{\sqrt{p^{\prime}(\tilde{\rho})}}{\tilde{\rho}} .
$$

CASE 2. For any $\rho_{1}, \rho\left(\xi_{0}-\right) \geq \rho_{1}>\tilde{\rho}$ there are points $\bar{\rho} \in\left(\tilde{\rho}, \rho_{1}\right)$ at which (3.19) holds as an equality.

It suffices to show that, for any monotonically decreasing sequence $\left\{\rho_{n}\right\}$ of the following two types converging to $\tilde{\rho}$ as $n \rightarrow \infty$,

$$
\lim _{n \rightarrow \infty} \frac{\Delta \bar{u}_{n}}{\Delta \rho_{n}}=\frac{\sqrt{p^{\prime}(\tilde{\rho})}}{\tilde{\rho}}
$$

where $\Delta \bar{u}_{n}=\bar{u}^{(1)}\left(\rho_{n}\right)-\bar{u}^{(1)}(\tilde{\rho}), \Delta \rho_{n}=\rho_{n}-\tilde{\rho}$.

Type 1: (3.19) holds as an equality at $\rho_{n}, n=2,3, \ldots$ In this case, we have, by Lemma 3.2, that

$$
\begin{gathered}
u_{n} \rho_{n}-\tilde{u}_{n} \tilde{\rho}_{n}-\xi_{0}\left(\rho_{n}-\tilde{\rho}\right)=0 \\
u_{n}^{2} \rho_{n}-\tilde{u}^{2} \tilde{\rho}-\xi_{0}\left(u_{n} \rho_{n}-m\left(\xi_{0}-\right)\right)+p\left(\rho_{m}\right)-p\left(\rho\left(\xi_{0}-\right)\right)
\end{gathered}
$$

where $u_{n}=\bar{u}^{(1)}\left(\rho_{n}\right)$. From (3.31) and (3.32), we can derive that the "left" derivative of $\bar{u}^{(1)}(\rho)$ at $(\tilde{u}, \tilde{\rho})$ is given by $(3.23)$.

Type 2: (3.19) hold as strict inequalities at $\rho_{n}, n=2,3, \ldots$.

Let $\left(\lambda_{n}, \bar{\lambda}_{n}\right)$ be the maximum intervals containing $\rho_{n}$ and in which (3.19) hold as strict inequalities. Then $\lambda_{n}, \bar{\lambda}_{n} \rightarrow \tilde{\rho}$ as $n \rightarrow \infty$. Consider

$$
\begin{aligned}
\frac{\Delta \bar{u}_{n}}{\Delta \rho_{n}}-\frac{\sqrt{p^{\prime}(\tilde{\rho})}}{\tilde{\rho}}= & \frac{\rho_{n}-\lambda_{n}}{\rho_{n}-\tilde{\rho}}\left(\frac{\bar{u}^{(1)}\left(\rho_{n}\right)-\bar{u}^{(1)}\left(\lambda_{n}\right)}{\rho_{n}-\lambda_{n}}-\frac{\sqrt{p^{\prime}(\tilde{\rho})}}{\tilde{\rho}}\right) \\
& +\frac{\lambda_{n}-\tilde{\rho}}{\rho_{n}-\tilde{\rho}}\left(\frac{\bar{u}^{(1)}\left(\lambda_{n}\right)-\bar{u}^{(1)}(\tilde{\rho})}{\lambda_{n}-\tilde{\rho}}-\frac{\sqrt{p^{\prime}(\tilde{\rho})}}{\tilde{\rho}}\right) .
\end{aligned}
$$

By the Case 2, Type 1, the second bracket approaches 0 as $n \rightarrow \infty$. The first bracket vanishes as $n \rightarrow \infty$ also in view of our claim for the Case 1 .

Analogously, we can prove that the "right" derivative of $\bar{u}(\rho)$ at $(\tilde{u}, \tilde{\rho})$ is given by (3.23). The proof for (c) is similar.

THEOREM 3.4. Let $\xi_{0}$ be a point of discontinuity of $(u(\xi), \rho(\xi))$ with $\rho\left(\xi_{0} \pm\right)>0$. Then

$$
\begin{aligned}
& u\left(\xi_{0}-\right)+S\left(u\left(\xi_{0}-\right), \rho\left(\xi_{0}-\right) ;+\right) \sqrt{p^{\prime}\left(\rho\left(\xi_{0}-\right) \geq \xi_{0}\right.} \\
& \geq u\left(\xi_{0}+\right)+S\left(u\left(\xi_{0}+\right), \rho\left(\xi_{0}+\right) ;-\right) \sqrt{p^{\prime}\left(\rho\left(\xi_{0}+\right)\right.} .
\end{aligned}
$$

Proof. Suppose that $P(s)$ is decreasing (increasing) from the right of $\left(u\left(\xi_{0}-\right)\right.$, $\left.\rho\left(\xi_{0}\right)\right)$. The proofs for other cases are similar. Then for $(\bar{u}, \bar{\rho}) \in C_{\xi_{0}}$ and close to $\left(u\left(\xi_{0}-\right), \rho\left(\xi_{0}-\right)\right)$ enough, we have, by Lemma 3.2, that

$$
\bar{u} \bar{\rho}-m\left(\xi_{0}-\right)-\xi_{0}\left(\bar{\rho}-\rho\left(\xi_{0}-\right)\right) \leq 0 .
$$


If we recall Theorem 3.3 and are careful about the sign of $\bar{\rho}-\rho\left(\xi_{0}-\right)$, we can obtain

$$
\xi_{0} \leq \frac{\bar{u} \bar{\rho}-m\left(\xi_{0}-\right)}{\bar{\rho}-\rho\left(\xi_{0}-\right)} \rightarrow u\left(\xi_{0}-\right)+S\left(u\left(\xi_{0}-\right), \rho\left(\xi_{0}-\right) ;+\right) \sqrt{p^{\prime}\left(\rho\left(\xi_{0}-\right)\right.}
$$

as $\bar{\rho} \rightarrow \rho\left(\xi_{0}-\right)$. Similarly, we can prove the other half of (3.34).

Let $\tau_{n}$ be the critical point (if there is any) for $u_{\epsilon_{n}}(\xi)$, or $\rho_{\epsilon_{n}}(\xi), n=1,2, \ldots$. By the uniform boundedness and Lemma 2.3, there is a subsequence of $\left\{\epsilon_{n}\right\}$, denoted by $\left\{\epsilon_{n}\right\}$ again, such that $\left(u_{\epsilon_{n}}\left(\tau_{n}\right), \rho_{\epsilon_{n}}\left(\tau_{n}\right)\right) \rightarrow\left(u_{0}, \rho_{0}\right)$ as $n \rightarrow \infty$. Clearly, $\left(u_{0}, \rho_{0}\right)$ lies on the base curve.

COROLlaRY 3.5. If $\rho_{\epsilon_{n}}(\xi)$ have critical minimum values at $\tau_{n}$ for $n$ sufficiently large and if $\left(u_{\epsilon_{n}}\left(\tau_{n}\right), \rho_{\epsilon_{n}}\left(\tau_{n}\right)\right) \rightarrow\left(u_{0}, \rho_{0}\right)$ as $n \rightarrow \infty$. Then

(a) $\left(u_{0}, \rho_{0}\right)=(u(\xi), \rho(\xi))$ for some $\xi \in \mathbb{R}$, if $\rho_{0}>0$.

(b) $\rho(\xi \pm)=0$ for some $\xi \in \mathbb{R}$, if $\rho_{0}=0$.

Proof. By Lemma 2.1, $u_{\epsilon_{n}}(\xi)$ are increasing functions so is $u(\xi)$. So, $u_{-} \leq u_{0} \leq$ $u_{+}$. Assume the contrary of the Corollary. Then there is a point of discontinuity $\xi_{0}$ of $(u(\xi), \rho(\xi))$ such that $u\left(\xi_{0}-\right)<u_{0}<u\left(\xi_{0}+\right)$ and $\rho\left(\xi_{0} \pm\right)>0$. By Theorem 3.4, we have

$$
u\left(\xi_{0}-\right)+\sqrt{p^{\prime}\left(\rho\left(\xi_{0}-\right)\right.} \geq \xi_{0} \geq u\left(\xi_{0}+\right)+\sqrt{p^{\prime}\left(\rho\left(\xi_{0}+\right)\right.}
$$

which is impossible.

Corollary 3.6. There is a $\delta>0$ which depends only on $p(\rho), \rho \pm$ such that $\xi_{0}$ is not a point of discontinuity of $(u(\xi), \rho(\xi))$ whenever $0<\rho\left(\xi_{0}-\right)<\delta$ or $0<\rho\left(\xi_{0}+\right)<\delta$ holds.

Proof. By Assumption $1, p^{\prime}(\rho)$ is increasing for $\rho \in\left(0, \delta_{1}\right)$. We can choose $0<\delta<\min \left(\delta_{1}, \rho_{-}, \rho_{+}\right)$such that

$$
0<p^{\prime}(\rho)<\min _{\rho \in\left(\delta, \rho^{*}\right]}\left(p^{\prime}(\rho)\right)
$$

for $0<\rho<\delta$, where $\rho^{*}=: \max \left(\rho_{-}, \rho_{+}\right)$.

If $\xi_{0}$ is a point of discontinuity of $(u(\xi), \rho(\xi))$ and $\rho\left(\xi_{0}-\right)$ or $\rho\left(\xi_{0}+\right)<\delta$, then $\rho_{\epsilon_{n}}(\xi)$ have critical minimum points and $u_{\epsilon_{n}}(\xi)$ are increasing function for $\epsilon_{n}$ small. By Theorem 3.4 and the proof of Corollary 3.5, either

$$
u\left(\xi_{0}-\right)-\sqrt{p^{\prime}\left(\rho\left(\xi_{0}-\right)\right.} \geq \xi_{0} \geq u\left(\xi_{0}+\right)-\sqrt{p^{\prime}\left(\rho\left(\xi_{0}+\right)\right.}
$$

where $\rho\left(\xi_{0}+\right)<\rho\left(\xi_{0}-\right), u\left(\xi_{0}+\right)>u\left(\xi_{0}-\right)$, or

$$
u\left(\xi_{0}-\right)+\sqrt{p^{\prime}\left(\rho\left(\xi_{0}-\right)\right.} \geq \xi_{0} \geq u\left(\xi_{0}+\right)+\sqrt{p^{\prime}\left(\rho\left(\xi_{0}+\right)\right.}
$$

where $\rho\left(\xi_{0}+\right)>\rho\left(\xi_{0}-\right), u\left(\xi_{0}+\right)>u\left(\xi_{0}-\right)$ holds. But either (3.37) or (3.38) implies that $p^{\prime}\left(\rho_{1}\right)<p^{\prime}\left(\rho_{2}\right)$ for some $\rho_{2}<\rho_{1}$ and $\rho_{2}<\delta$, which contradicts our choice of $\delta$.

We say that a function $f(\xi)$ is strictly increasing (or decreasing) from the left at $\xi_{0} \in \mathbb{R}$ if $f(\xi)<$ (or $>$ ) $f\left(\xi_{0}-\right)$ for $\xi \in\left(\xi_{0}-\tau, \xi_{0}\right)$ for some $\tau>0$. We say $f(\xi)$ is strictly monotone from the left at $\xi_{0}$ if it is either strictly increasing or strictly decreasing from the left at $\xi_{0}$. A similar thing can be said for the "right" at $\xi_{0}$. 
THEOREM 3.7. (a) If $u_{\epsilon_{n}}(\xi)$ or $\rho_{\epsilon_{n}}(\xi)$ is strictly monotone from the left at $\xi_{0} \in \mathbb{R}$ then

$$
\begin{gathered}
\xi_{0}=u\left(\xi_{0}-\right)+S\left(u\left(\xi_{0}-\right), \rho\left(\xi_{0}-\right) ;-\right) \sqrt{p^{\prime}\left(\rho\left(\xi_{0}-\right)\right)}, \\
\frac{d \bar{u}(\rho)}{d^{(-)} \rho}=S\left(u\left(\xi_{0}-\right), \rho\left(\xi_{0}-\right) ;-\right) \frac{\sqrt{p^{\prime}\left(\rho\left(\xi_{0}-\right)\right)}}{\rho\left(\xi_{0}-\right)} .
\end{gathered}
$$

(b) If $u(\xi)$ or $\rho(\xi)$ is strictly monotone from the right at $\xi_{0} \in \mathbb{R}$, then

$$
\begin{gathered}
\xi_{0}=u\left(\xi_{0}+\right)+S\left(u\left(\xi_{0}+\right), \rho\left(\xi_{0}+\right) ;+\right) \sqrt{p^{\prime}\left(\rho\left(\xi_{0}+\right)\right)}, \\
\frac{d \bar{u}(\rho)}{d^{(+)} \rho}=S\left(u\left(\xi_{0}+\right), \rho\left(\xi_{0}+\right) ;+\right) \frac{\sqrt{p^{\prime}\left(\rho\left(\xi_{0}+\right)\right)}}{\rho\left(\xi_{0}+\right)} .
\end{gathered}
$$

Proof. We prove the case when $\rho(\xi)$ is strictly increasing from the left of $\xi_{0}$ only. Without loss of generality, we can assume that $(u(\xi), \rho(\xi))$ is on the curve $u=\bar{u}^{(1)}(\rho)$. The proofs for the other cases are similar. We shall prove that

$$
\lim _{n \rightarrow \infty} \frac{\Delta \bar{u}_{n}}{\Delta \rho_{n}}=S\left(u\left(\xi_{0}-\right), \rho\left(\xi_{0}-\right) ;-\right) \frac{\sqrt{p^{\prime}\left(\rho\left(\xi_{0}-\right)\right)}}{\rho\left(\xi_{0}-\right)}
$$

for any $\left\{\rho_{n}\right\}$ which increases to $\rho\left(\zeta_{0}-\right)$ as $n \rightarrow \infty$, where $\Delta \rho_{n}=\rho_{n}-\rho\left(\xi_{0}-\right), \Delta \bar{u}_{n}=$ $\bar{u}^{(1)}\left(\rho_{n}\right)-u\left(\xi_{0}-\right)$. It suffices to show the assertion for the following two cases:

CASE 1. $\left(\bar{u}^{(1)}\left(\rho_{n}\right), \rho_{n}\right)=\left(u\left(\xi_{n}+\right), \rho\left(\xi_{n}+\right)\right)$ for some $\xi_{n}<\xi_{0}$. Integrating (1.3) from $\xi_{n}$ to $\xi$, we obtain

$$
\begin{gathered}
\frac{\Delta_{n} m}{\Delta_{n} \rho}-\xi_{0}-\frac{1}{\Delta_{n} \rho} \int_{\xi_{n}}^{\xi_{0}}\left(\rho\left(\xi_{0}-\right)-\rho(\zeta)\right) d \zeta=0, \\
\frac{\Delta_{n}\left(u^{2} \rho+p(\rho)\right)}{\Delta_{n} \rho}-\xi_{0} \frac{\Delta_{n} m}{\Delta_{n} \rho}+\frac{1}{\Delta_{n} \rho} \int_{\xi_{n}}^{\xi_{0}}\left(m\left(\xi_{0}-\right)-m(\zeta)\right) d \zeta=0,
\end{gathered}
$$

where $\Delta_{n} f=f\left(\xi_{n}\right)-f\left(\xi_{0}-\right)$. Letting $n \rightarrow \infty$ in (3.44), (3.45) and recalling Lemma 2.3 , we deduce that

$$
\begin{gathered}
\rho\left(\xi_{0}-\right) \lim _{n \rightarrow \infty} \frac{\Delta_{n} u}{\Delta_{n} \rho}+u\left(\xi_{0}-\right)-\xi_{0}=0 \\
u^{2}\left(\xi_{0}-\right)+p^{\prime}\left(\rho\left(\xi_{0}-\right)\right)-\xi_{0} u\left(\xi_{0}-\right)+\left(2 m\left(\xi_{0}-\right)-\xi_{0} \rho\left(\xi_{0}-\right)\right) \lim _{n \rightarrow \infty} \frac{\Delta_{n} u}{\Delta_{n} \rho}=0 .
\end{gathered}
$$

From these two equations, it follows that

$$
\begin{gathered}
\lim _{n \rightarrow \infty} \frac{\Delta_{n} u}{\Delta_{n} \rho}= \pm \frac{\sqrt{p^{\prime}\left(\xi_{0}-\right)}}{\rho\left(\xi_{0}-\right)}, \\
\xi_{0}=u\left(\xi_{0}-\right) \pm \sqrt{p^{\prime}\left(\rho\left(\xi_{0}-\right)\right.} .
\end{gathered}
$$

Thus (3.39-3.42) are valid in this case.

CASE 2. $\left(\bar{u}^{(1)}\left(\rho_{n}\right), \rho_{n}\right) \neq(u(\xi+), \rho(\xi+))$ for an $\xi \in \mathbb{R}, n=1,2, \ldots$ 
Then, there are points, $\xi_{n}$, of discontinuity of $(u(\xi), \rho(\xi))$ such that $\left(\bar{u}^{(1)}\left(\rho_{n}\right), \rho_{n}\right)$ $\in C_{\xi_{n}}$. Since $\rho_{n}$ strictly increase to $\rho\left(\xi_{0}-\right)$, we expect that $\rho\left(\xi_{n} \pm\right) \rightarrow \rho\left(\xi_{0}-\right)-$ as $n \rightarrow \infty$. We have

$$
\begin{aligned}
\frac{\Delta \bar{u}_{n}}{\Delta \rho_{n}}-\frac{\sqrt{p^{\prime}\left(\rho\left(\xi_{0}-\right)\right.}}{\rho\left(\xi_{0}-\right)}= & \frac{\rho_{n}-\rho\left(\xi_{n}+\right)}{\Delta \rho_{n}}\left(\frac{\bar{u}^{(1)}\left(\rho_{n}\right)-u\left(\xi_{n}+\right)}{\rho_{n}-\rho\left(\xi_{0}-\right)}-\frac{\sqrt{p^{\prime}\left(\rho\left(\xi_{0}-\right)\right.}}{\rho\left(\xi_{0}-\right)}\right) \\
& +\frac{\rho\left(\xi_{n}+\right)-\rho\left(\xi_{0}-\right)}{\Delta \rho_{n}}\left(\frac{u\left(\xi_{n}+\right)-u\left(\xi_{0}-\right)}{\rho\left(\xi_{n}+\right)-\rho\left(\xi_{0}-\right)}-\frac{\sqrt{p^{\prime}\left(\rho\left(\xi_{0}-\right)\right.}}{\rho\left(\xi_{0}-\right)}\right) .
\end{aligned}
$$

By Case 1, the second bracket approaches to 0 as $n \rightarrow \infty$. By Lemma 3.3, we know that the portion $C_{\xi_{n}}$ of the base curve is differentiable for $n$ large and hence

$$
\frac{\bar{u}^{(1)}\left(\rho_{n}\right)-u\left(\xi_{n}+\right)}{\rho_{n}-\rho\left(\xi_{n}+\right)}=\left.\frac{d U(s)}{d P(s)}\right|_{s=s_{n}}=\left.\frac{d \hat{u}(\zeta)}{d \hat{\rho}(\zeta)}\right|_{\zeta=0}
$$

for some $s_{n} \in \mathbb{R}$, where $(\hat{u}(\zeta), \hat{\rho}(\zeta))$ are the solutions of (3.6) with

$$
(\hat{u}(0), \hat{\rho}(0))=\left(U\left(s_{n}\right), P\left(s_{n}\right)\right)
$$

Recalling the claim we proved in the proof of Lemma 3.3, we have that

$$
\min _{\zeta \in \mathbb{R}} a_{ \pm}(\zeta) \leq \frac{d \hat{u}(\zeta)}{d \hat{\rho}(\zeta)} \leq \max _{\zeta \in \mathbb{R}} a_{ \pm}(\zeta)
$$

where

$$
a_{ \pm}(\zeta)=: \frac{-\hat{\rho}(\zeta) \pm \sqrt{\hat{\rho}^{\prime 2}(\zeta)+\hat{\rho}^{2}(\zeta) p^{\prime}(\hat{\rho}(\zeta))}}{\hat{\rho}^{2}(\zeta)}
$$

where $\hat{\rho}^{\prime}(\zeta)$ is given by $(3.6 \mathrm{a})$ :

$$
\hat{\rho}^{\prime}(\zeta)=\hat{m}(\zeta)-m\left(\xi_{0}-\right)-\xi_{0}\left(\hat{\rho}(\zeta)-\rho\left(\xi_{0}-\right)\right) .
$$

Noting that $\left(u\left(\xi_{n} \pm\right), \rho\left(\xi_{n} \pm\right)\right) \rightarrow\left(u\left(\xi_{0}-\right), \rho\left(\xi_{0}-\right)\right)$ as $n \rightarrow \infty$, we can easily see that (3.51) approaches to $\sqrt{p^{\prime}\left(\rho\left(\xi_{0}-\right)\right.} / \rho\left(\xi_{0}-\right)$ as $n \rightarrow \infty$. Thus the right hand side of (3.50) vanishes as $n \rightarrow \infty$.

Corollary 3.8. (a) The solution $(u(\xi), \rho(\xi))$ have no constant states other than $\left(u_{-}, \rho_{-}\right),\left(u_{+}, \rho_{+}\right)$and possibly $\left(u_{0}, \rho_{0}\right)$.

(b) Let $\xi_{0}$ be a point of discontinuity of $(u(\xi), \rho(\xi))$. If $\left(u\left(\xi_{0}-\right), \rho\left(\xi_{0}-\right)\right)$ (or $\left.\left(u\left(\xi_{0}+\right), \rho\left(\xi_{0}+\right)\right)\right) \neq\left(u_{0}, \rho_{0}\right),\left(u_{ \pm}, \rho_{ \pm}\right)$, then $\xi_{0}$ is a contact discontinuity from the left (or right).

Proof. (a) Suppose that $(\bar{u}, \bar{\rho}) \neq\left(u_{ \pm}, \rho_{ \pm}\right),\left(u_{0}, \rho_{0}\right)$ and

$$
(u(\xi), \rho(\xi))=(\bar{u}, \bar{\rho})
$$

for $\xi \in\left(\xi_{1}, \xi_{2}\right)$, where $\left(\xi_{1}, \xi_{2}\right)$ is a maximum interval in which the above equation holds. Then $S(\bar{u}, \bar{\rho} ;+)=S(\bar{u}, \bar{\rho} ;-)=: S$. By Theorem 3.4, 3.5, we have that

$$
\bar{u}+S \sqrt{p^{\prime}(\bar{\rho})} \geq \xi_{2}>\xi_{1} \geq \bar{u}+S \sqrt{p^{\prime}(\bar{\rho})}
$$

which is a contradiction.

(b) We can prove this by combining Theorem 3.4 and 3.7. 
LemMA 3.9. Let $\xi_{0}$ be a point of discontinuity of $(u(\xi), \rho(\xi))$. Then

$$
\left|\frac{u\left(\xi_{0}+\right)-u\left(\xi_{0}-\right)}{\rho\left(\xi_{0}+\right)-\rho\left(\xi_{0}-\right)}\right| \leq \frac{\max \left(\sqrt{p^{\prime}\left(\rho\left(\xi_{0}-\right)\right.}, \sqrt{\left.p^{\prime}\left(\rho\left(\xi_{0}+\right)\right)\right)}\right.}{\min \left(\rho\left(\xi_{0}-\right), \rho\left(\xi_{0}+\right)\right)} .
$$

Proof. One of the Rankine-Hugoniot conditions is

$$
\xi_{0}\left(\rho\left(\xi_{0}+\right)-\rho\left(\xi_{0}-\right)\right)=m\left(\xi_{0}+\right)-m\left(\xi_{0}-\right) .
$$

We rewrite it as

$$
\frac{\Delta u}{\Delta \rho}=\frac{\xi_{0}-u\left(\xi_{0}-\right)}{\rho\left(\xi_{0}+\right)}=\frac{\xi_{0}-\rho\left(\xi_{0}+\right)}{\rho\left(\xi_{0}-\right)}
$$

where $\Delta u=u\left(\xi_{0}+\right)-u\left(\xi_{0}-\right), \Delta \rho=\rho\left(\xi_{0}+\right)-\rho\left(\xi_{0}-\right)$. Noting the inequalities (3.34), we obtain that

$$
S\left(u\left(\xi_{0}+\right), \rho\left(\xi_{0}+\right) ;-\right) \frac{\sqrt{p^{\prime}\left(\rho\left(\xi_{0}+\right)\right)}}{\rho\left(\xi_{0}-\right)} \leq \frac{\Delta u}{\Delta \rho} \leq S\left(u\left(\xi_{0}-\right), \rho\left(\xi_{0}-\right) ;+\right) \frac{\sqrt{p^{\prime}\left(\rho\left(\xi_{0}-\right)\right)}}{\rho\left(\xi_{0}+\right)}
$$

and hence the result.

THEOREM 3.10. Suppose $u_{+}>u_{-}$, and $\rho^{*}=: \max \left(\rho_{+}, \rho_{-}\right), \rho^{*}=: \max \left(\rho_{+}, \rho_{-}\right)$.

(a) If

$$
u_{+}-u_{-}>\frac{\max _{\rho \in\left[\rho_{*}, \rho^{*}\right]}\left(p^{\prime}(\rho)\right)}{\rho *}\left|\rho_{+}-\rho_{-}\right|,
$$

then $\rho_{\epsilon_{n}}(\xi)$ must have critical points which are minimum points, for $\epsilon$ small enough.

(b) If $\int_{0}^{\delta} \sqrt{p^{\prime}(\rho)} / \rho d \rho<+\infty$ and

$$
u_{+}-u_{-}>2 \int_{0}^{\delta} \frac{\sqrt{p^{\prime}(\rho)}}{\rho} d \rho+\frac{\max _{\rho \in\left[\delta, \rho^{*}\right]}\left(p^{\prime}(\rho)\right)}{\delta}\left(\rho_{+}+\rho_{-}-2 \delta\right),
$$

where $\delta$ is the constant in Corollary 3.6, then the solution of $(1.1),(u(\xi), \rho(\xi))$ possesses a vacuum state. Furthermore, the solution can have at most one vacuum state. This vacuum state is necessarily a rarefaction vacuum state.

Proof. We prove (b) only. The proof for (a) is similar. Since (3.57) implies (3.56), $\rho_{\epsilon_{n}}(\xi)$ have critical points $\tau_{n}$ which are minimum points of $\rho_{\epsilon_{n}}(\xi)$. We denote $\left(u_{0}, \rho_{0}\right)=: \lim _{n \rightarrow \infty}\left(u_{\epsilon_{n}}\left(\tau_{n}\right), \rho_{\epsilon_{n}}\left(\tau_{n}\right)\right)$. By Corollary $3.5(\mathbf{b})$, it suffices to show that $\rho_{0}=0$. Assume the contrary, i.e., $\rho_{0}>0$.

We define that

$$
\begin{gathered}
\Omega_{1, c}=:\left\{\rho \in\left[\rho_{0}, \rho_{-}\right] \mid(u(\xi-), \rho(\xi-))\right. \\
\text { or } \left.(u(\xi+), \rho(\xi+))=\left(\bar{u}^{(1)}(\rho), \rho\right) \text { for some } \xi \in \mathbb{R}\right\}, \\
\Omega_{1, \text { disc }}=:\left[\rho_{0}, \rho_{-}\right] \backslash \Omega_{1, c}, \\
\Omega_{2, c}=:\left\{\rho \in\left[\rho_{0}, \rho_{+}\right] \mid(u(\xi-), \rho(\xi-))\right. \\
\text { or } \left.(u(\xi+), \rho(\xi+))=\left(\bar{u}^{(2)}(\rho), \rho\right) \text { for some } \xi \in \mathbb{R}\right\}, \\
\Omega_{2, \text { disc }}=:\left[\rho_{0}, \rho_{+}\right] \backslash \Omega_{2, c} .
\end{gathered}
$$




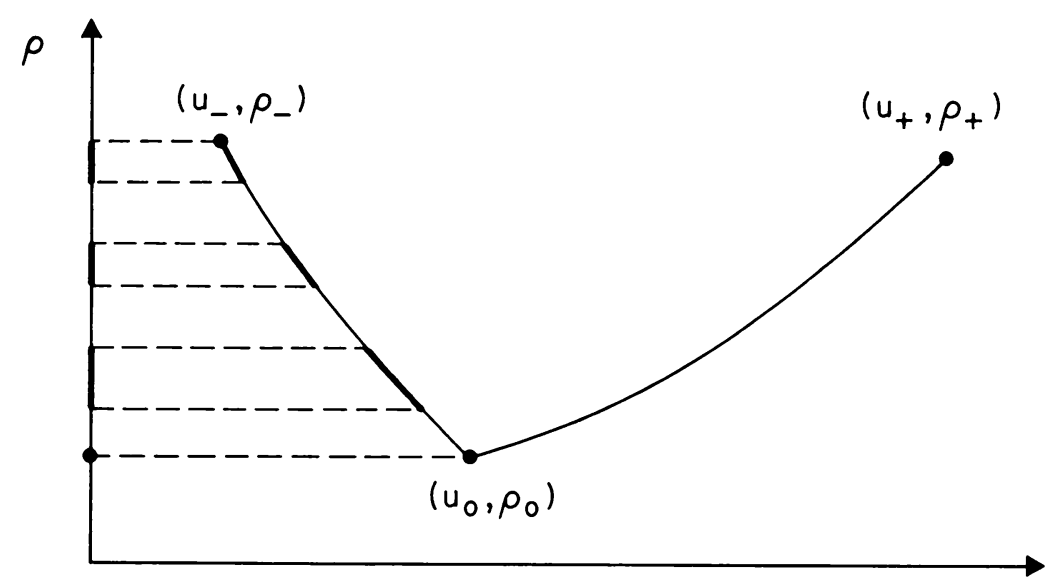

u

FIG. 4. The base curve when $\rho$ has an extreme value $\rho_{0}>0$, which is a minima. The thickened part on the base curve is the set of points $(u(\xi \pm), \rho(\xi \pm))$ on the branch $u=\bar{u}^{(1)}(\rho)$. The darkened part of the $\rho$-axis is $\Omega_{1, c}$.

If $\rho_{0}>0$, then

It is easy to see that

$$
\begin{aligned}
u_{+}-u_{-}= & \int_{\rho_{0}}^{\rho_{+}} \frac{d \bar{u}^{(2)}(\rho)}{d \rho} d \rho-\int_{\rho_{0}}^{\rho_{-}} \frac{d \bar{u}^{(1)}(\rho)}{d \rho} d \rho \\
= & \int_{\Omega_{2, c}} \frac{d \bar{u}^{(2)}(\rho)}{d \rho} d \rho+\int_{\Omega_{2, \text { disc }}} \frac{d \bar{u}^{(2)}(\rho)}{d \rho} d \rho \\
& -\int_{\Omega_{1, c}} \frac{d \bar{u}^{(1)}(\rho)}{d \rho} d \rho-\int_{\Omega_{1, \text { disc }}} \frac{d \bar{u}^{(1)}(\rho)}{d \rho} d \rho .
\end{aligned}
$$

$\Omega_{1, \text { disc }}=\bigcup\left\{(\rho(\xi+), \rho(\xi-)) \mid \rho(\xi+) \neq \rho(\xi-), u(\xi-)=\bar{u}^{(1)}(\rho(\xi-))\right.$ for some $\left.\xi \in \mathbb{R}\right\}$.

Then, for the last term in (3.58), we have

$$
\begin{aligned}
-\int_{\Omega_{1, \text { disc }}} \frac{d \bar{u}^{(1)}(\rho)}{d \rho} d \rho & =\sum_{\xi \in \mathbb{R}_{1, \text { disc }}} \int_{\rho(\xi+)}^{\rho(\xi-)} \frac{d \bar{u}^{(1)}(\rho)}{d \rho} d \rho \\
& =\sum_{\xi \in \mathbb{R}_{1, \text { disc }}} \frac{u(\xi-)-u(\xi+)}{\rho(\xi-)-\rho(\xi+)}(\rho(\xi-)-\rho(\xi+))
\end{aligned}
$$

where $\mathbb{R}_{1, \text { disc }}=:\left\{\xi \in \mathbb{R} \mid \rho(\xi-) \neq \rho(\xi+), u(\xi-)=\bar{u}^{(1)}(\rho(\xi-)\}\right.$. From Lemma 3.9 and Corollary 3.6, we derive that

$$
\begin{aligned}
\left|\int_{\Omega_{k, \text { disc }}} \frac{d \bar{u}^{(k)}(\rho)}{d \rho} d \rho\right| & \leq \sum_{\xi \in \in_{k, \text { disc }}} \frac{1}{\delta} \max _{\rho \in\left[\delta, \rho_{-}\right]}\left(\sqrt{p^{\prime}(\rho)}\right)(\rho(\xi-)-\rho(\xi+)) \\
& \leq \frac{1}{\delta} \max _{\rho \in\left[\delta, \rho^{*}\right]}\left(\sqrt{p^{\prime}(\rho)}\right) \mu\left(\Omega_{k, \text { disc }}\right)
\end{aligned}
$$


for $k=1,2$, where $\mu(E)$ is the Lebesgue measure of the set $E \subset \mathbb{R}$. Employing Theorem 3.7 and Corollary 3.6, we deduce that

$$
\begin{aligned}
\left|\int_{\Omega_{k, c}} \frac{d \bar{u}^{(k)}(\rho)}{d \rho} d \rho\right| & \leq \int_{0}^{\delta}\left|\frac{d \bar{u}^{(k)}(\rho)}{d \rho}\right| d \rho+\int_{\left[\delta, \rho^{*}\right] \cap \Omega_{k, c}}\left|\frac{d \bar{u}^{(k)}(\rho)}{d \rho}\right| d \rho \\
& \leq \int_{0}^{\delta} \frac{\sqrt{p^{\prime}(\rho)}}{\rho} d \rho+\frac{1}{\delta} \max _{\rho \in\left[\delta, \rho^{*}\right]} \sqrt{p^{\prime}(\rho)} \mu\left(\left[\delta, \rho^{*}\right] \cap \Omega_{k, c}\right)
\end{aligned}
$$

for $k=1,2$. Combining (3.58), (3.60) and (3.61), we obtain

$$
\begin{aligned}
u_{+}-u_{-} \leq & 2 \int_{0}^{\delta} \frac{\sqrt{p^{\prime}(\rho)}}{\rho} d \rho \\
& +\sum_{k=1,2}\left(\frac{1}{\delta} \max _{\rho \in\left[\delta, \rho_{-}\right]}\left(\sqrt{p^{\prime}(\rho)}\right) \mu\left(\Omega_{k, \text { disc }}\right)+\frac{1}{\delta} \max _{\rho \in\left[\delta, \rho^{*}\right]} \sqrt{p^{\prime}(\rho)} \mu\left(\left[\delta, \rho^{*}\right] \cap \Omega_{k, c}\right)\right) \\
\leq & 2 \int_{0}^{\delta} \frac{\sqrt{p^{\prime}(\rho)}}{\rho} d \rho+\frac{1}{\delta} \max _{\rho \in\left[\delta, \rho^{*}\right]} \sqrt{p^{\prime}(\rho)}\left(\rho_{+}+\rho_{-} 2 \delta\right) .
\end{aligned}
$$

Thus (3.57) is violated.

The following is a summary of my results: The solution of $(1.1)$ by the limiting viscosity approach given by [17] lies on a base curve in $(u, \rho)$-plane which is differentiable with respect to $u$ or $\rho$ except possibly at one of the extreme points of $u$ or $\rho$. The solution consists of two wave fans: a wave fan of the first kind with $\left(u_{-}, \rho_{-}\right)$on its left and a wave fan of the second kind with $\left(u_{+}, \rho_{+}\right)$on its right, separated by either the constant state $\left(u_{0}, \rho_{0}\right)$ or a shock. We recall that a wave fan is said of first (second) kind if it consists of 1-shocks (2-shocks) alternating with 1 -simple waves (2-simple waves) so that a shock adjacent to a wave on one of its sides is a contact discontinuity from this side. A $\xi \in \mathbb{R}$ is in the first wave fan (or the second one) if the slope of the base curve at $(u(\xi \pm), \rho(\xi \pm))$ is negative (positive). If $\rho(\xi+)$ or $\rho(\xi-)<\delta$, then $\xi$ is a point of continuity of $(u(\xi), \rho(\xi))$. Further, the solution has at most one vacuum state which is necessarily, a rarefaction one in the sense of Definition 1.2. If the initial conditions satisfy (3.57), the solution must possess a vacuum state.

Acknowledgment. I would like to thank Professor M. Slemrod for his valuable suggestions and comments.

\section{REFERENCES}

[1] R. DiPerna, Existence in the large nonlinear hyperbolic conservation laws, Arch. Rational Mech. Anal. 52, 244-257 (1973)

[2] J. Greenberg, On the interaction of shocks and simple waves of the same family, Arch. Rational Mech. Anal. 37, 136-160 (1970)

[3] T.-P. Liu, Shock waves in the nonisentropic gas flow, J. Differential Equations 22, 442-452 (1976)

[4] T.-P. Liu, Solution in the large for the equations of nonisentropic gas dynamics, Indiana Univ. Math. J. 26, 147-177 (1977)

[5] T.-P. Liu, The deterministic version of the Glimm scheme, Comm. Math. Phys. 57, 135-148 (1977) 
[6] T. Nishida and J. Smoller, Solutions in the large for some nonlinear hyperbolic conservation laws, Comm. Pure Appl. Math. 26, 183-200 (1973)

[7] R. Smith, The Riemann problem in gas dynamics, Trans. Amer. Math. Soc. 249, 1-30 (1979)

[8] Zhang Tong and Guo Yu-Fa, A class of initial value problems for systems of aerodynamic equations, Acta. Math. Sinica 15 386-396 (1965), English translation in Chinese Math. 7, 90-101 (1965)

[9] T.-P. Liu and J. Smoller, On the vacuum state for the isentropic gas dynamics equations, Advances in Appl. Math. 1, 345-359 (1980)

[10] C. M. Dafermos, Solution of the Riemann problem for a class of hyperbolic conservation laws by the viscosity method, Arch. Rational Mech. Anal. 52, 1-9 (1973)

[11] C. M. Dafermos, Structure of the solutions of the Riemann problem for hyperbolic conservation laws, Arch. Rational Mech. Anal. 53, 203-217 (1974)

[12] C. M. Dafermos and R. J. DiPerna, The Riemann problem for certain classes of hyperbolic systems of conservation laws, J. Differential Equations 20, 90-114 (1976)

[13] C. M. Dafermos, Admissible wave fans in nonlinear hyperbolic system, Arch. Rational Mech. Anal. 106, 243-260 (1989)

[14] A. S. Kalasnikov, Construction of generalized solutions of quasi-linear equations of first order without convexity conditions as limits of solutions of parabolic equations with a small parameter, Dokl. Adak. Nauk. SSSR 127, 27-30 (1959)

[15] B. L. Keyfitz and H. C. Kranzer, A viscosity approximation to a system of conservation laws with no classical Riemann solution, to appear in Proc. of Int. Conf. on Hyperbolic Problems, Bordeanux, (1989)

[16] M. Slemrod, A limiting "viscosity" approach to the Riemann problem for materials exhibiting change of phase, Arch. Rational Mech. Anal. 105, 327-365 (1989)

[17] M. Slemrod and A. Tzavaras, A limiting viscosity approach for the Riemann problem in isentropic gas dynamics, preprint (1989)

[18] V. A. Tupciev, On the method of introducing viscosity in the study of problems involving the decay of discontinuity, Dokl. Akad. Nauk. SSSR 211, 55-58 (1973) 